\title{
STATE OF THE ART SURVEY ON USING ROBOTS IN OIL AND GAS INDUSTRY
}

\author{
Ahmed Sachet Hashim ${ }^{1}$, Bogdan Grămescu ${ }^{2}$, Constantin Niţu ${ }^{3}$ \\ 1,2,3 University POLITEHNICA of Bucharest \\ 313, Splaiul Independenţei, 060042-Bucharest, Romania \\ ahmedhashim774@yahoo.com
}

\begin{abstract}
The objective of this study regards the using of robotics to improve the oil and gas industry. The study is necessary because, since the previous years, researchers and scientists have increased interest in finding the best means to protect oil and gas pipelines that are not replaceable, in order to detect the most important defects that occurs in oil and gas pipes. The most important defects, such as cracks, corrosion and leakage, occurs as a result of problems in welding areas, high temperature, aging in the infrastructure of pipelines and others. In order to solve the problem, the robot sends accurate data on the location and severity of the defects that occur in the pipes to the worker, for the purpose of early maintenance on the pipelines, to reduce risks in the future.
\end{abstract}

Keywords: Robotics, Oil Industry, Gas Industry, Pipes Inspection.

\section{Introduction}

The oil and gas industry keeps on blasting in the following couple of decades [1]. The requirements for a crude oil extraction plant become more complex, and the cost is growing. That's why the oil and gas industry is looking for solutions with high performances, but lower costs. It is additionally searching for approaches to cut costs and enhance fabricating efficiency and quality, but also to look for lower-cost crude materials. One problem is the high cost of the representative's compensation and advantages. The oil and gas organizations are searching for new advances to decrease the cost of work. One solution is to use programmed robot for repetitive works. For example, in order to ensure efficient pipelines performance, the pipelines need to have a routine monitoring, maintenance and repairing activities. However, due to the nature of pipelines that are long it is hard to accomplish the tasks. Human work for the cleaning and maintenance process is not efficient, safe and economical. There is a strong demand for robots that are able to inspect, to clean and repair the pipelines. With a specific end goal to accomplish the difficulties of mechanical technology and extend the use of robots in the oil and gas industry, overview and examination of the oil and gas industry operations are fundamental. For example, it is very important to find a problem before something wrong to happen. The utilization of programmed robot to check the pipelines to look for welding issues or bends, valves problems, to recognize inside deformities utilizing ultrasound is the most efficient way. The information is send to the specialists and they will decide the level of trust for a pipe. The main reason of this paper is a study about the state of art in using robots in oil and gas industry and talk about future research openings in the field of oil and gas in order to increase the efficiency and keep any risk at very low level.

\section{What Is A Robot?}

A robot is a machine or a delicate gadget with the effectiveness which resembles an individual intended to perform perplexing and diverse undertakings (as strolling or talking). It is a work consequently under the control of a PC with speed and precision. Robots are diverse structures relying upon the work allocated to him. The robot is a branch of the building that incorporates the idea, the plan, production and operation of robots. This meddles with the field of hardware and software engineering, man-made brainpower, mechatronics, nanotechnology, and bioengineering. The word 'robot' was first used to denote a fictional humanoid in a 1920 play R.U.R. by the Czech writer, Karel Čapek. The rules of robotics, that must drive the robots and keen machines, are:

1 - Robots must not hurt people.

2 - Robots must take after directions from people without disregarding Article 1.

3 - Robots must secure themselves without abusing different tenets.

The robotic equipment involves a mechanical structure, an electronic circuit, actuators, sensors, and a program that make the robot "smart". The 
mission of a robot is to supplant people in unsafe, hazardous areas or in the case of a repetitive work [2].

\section{Technical Robotics Terms}

\section{1 - Speed}

Speed is the amount of distance per unit time at which the robot can move, usually specified in Inches per second or meters per second. The speed is usually specified at a specific load or assuming that the robot is carrying a fixed weight. Actual speed may vary depending upon the weight carried by the robot.

\section{2 - Load Bearing Capacity}

Load bearing capacity is the maximum weightcarrying capacity of the robot. Serial robots that carry large weights, but must still be precise, are heavy and expensive, with poor (low) payload-toweight ratios.

\section{3 - Accuracy}

Accuracy is the ability of a robot to go to the specified position without making a mistake. It is impossible to position a machine exactly. Accuracy is therefore defined as the ability of the robot to position itself to the desired location with the minimal error (usually 0.001 inches).

\section{4 - Repeatability}

Repeatability is the ability of a robot to repeatedly position itself when asked to perform a task multiple times. Accuracy is an absolute concept, repeatability is relative. Note that a robot that is repeatable may not be very accurate. Likewise, an accurate robot may not be repeatable.

\section{5 - Precision}

Accuracy is the "purity" that makes a sensor most valuable than other. For example, a sensor that reads 2.1178 is more accurate than a sensor that reads the 2.1 for the same physical variable. Accuracy could be associated with the increase in the number of digits [3].

\section{Specific Robot Classification}

\section{From the autonomy point of view, the robots can be classified as: \\ 1- Non-autonomous robots \\ 2-Semi-autonomous robots \\ 3- Fully autonomous robots}

\subsection{Non-autonomous robots}

The non autonomous robot is in fact a remote machine, working under a human command to check a hazardous area, where the driver cannot go or enter (unsafe zones). The robot works remotely, for the most part of the time, sends and receives control signs through a fastened and secure link. For example, in the case of cleaning pipes, the robot sends pictures from a camera mounted on it, and the user must react with specific commands. These kinds of robots are normally utilized as a part of a monitoring system that involves human driver. Some examples are the robots from RAUSCH Electronics in the USA (Figure 1).

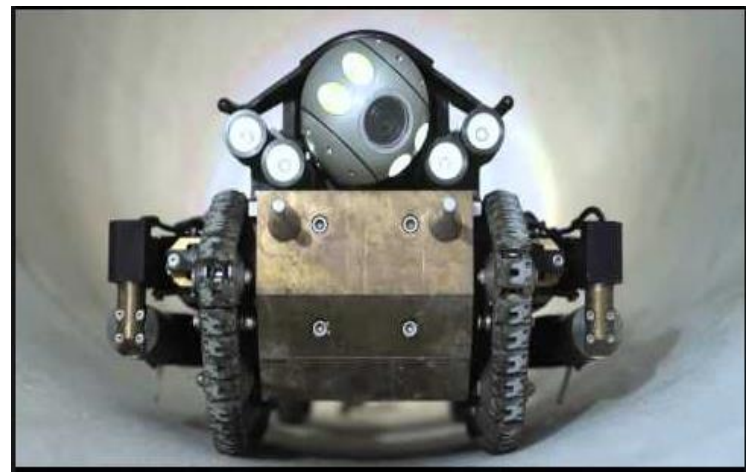

Fig. 1. Example of non-autonomous robot

\subsection{Semi-autonomous robots}

A semi-autonomous robot is a robotic structure, which has a unit to enable the robot to perform the work, which is usually a program on the modules that prepared the starting process. After that the control has to be carried out by the human factor. This makes the robot semi-autonomous. Some examples could be (a) Carew (b) PIPA (Figure 2).

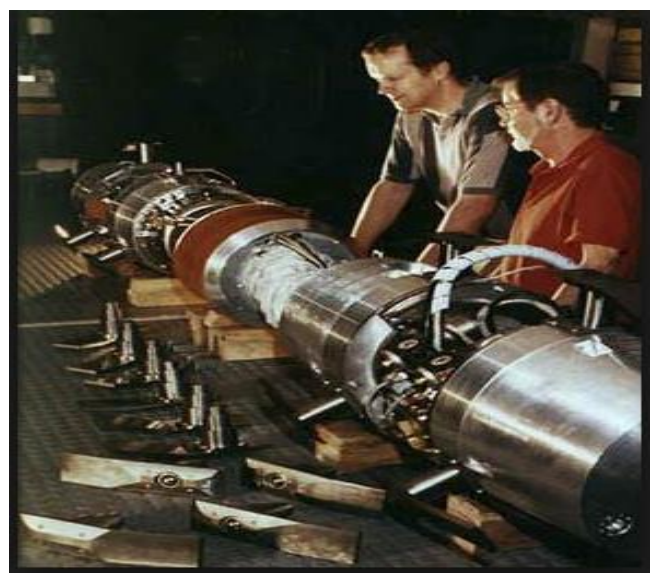

Fig. 2. Example of semi-autonomous robots

\subsection{Fully autonomous robots}

The robot has a program that allows it to be independent. It has to be prepared for all circumstances in the pipeline. For these robots, the control signals are transmitted by means of a remote connection. The control program, which ordinarily keeps running in with a human worker, sends commands to the robot. The robot works alone, but from time to time there are some status messages between robot and a human inspector or a computerized device, through a radio connection. 
If there are some corrections, there are sent to the robot, so it is adjusted to these changes, taking additionally moves to be made by the controllers automated. Some examples of completely autonomous robots are (a) Macro (Figure 3) and (b) Kentaro.

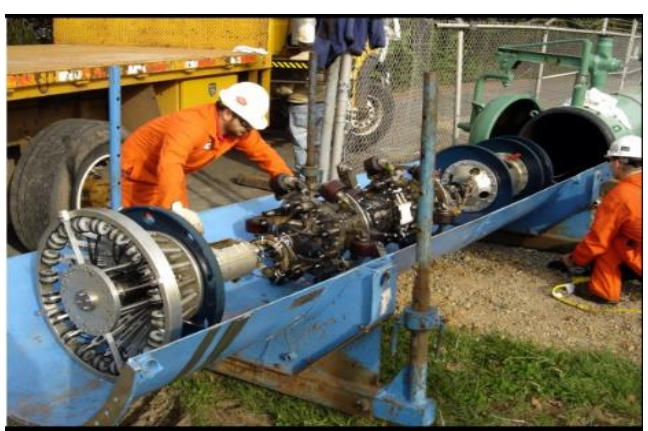

Fig. 3. Example of fully autonomous robot

\section{Robot Classification in the Case of Oil and Gas Pipelines}

The robot can work inside and outside oil and gas pipelines. Robots that work outside the tubes are less adaptable than the robots that operate inside the tubes. To address the above difficulty, these types of robots will be referred to their various uses with the aim of being reasonable to operate in these conditions, regardless of whether they are above ground or underground.

Those types of robots are:
a. Pig type robots
b. Wheel type robots
c. Caterpillar-type robots
d. Wall -press robots
e. Inch worm type robots
f. Screw type robots (helical type robots)

\section{a. Pig type robots}

This is a standout amongst the most well-known business robots, commonly utilized when there is a sufficient stream of liquid in the pipeline, which can adequately help to push the robot with the nonstop stream in the pipeline. This kind of robot is used in the case of large pipelines.

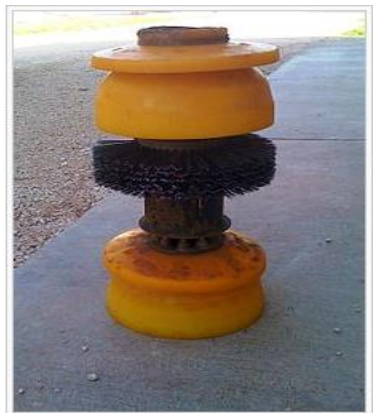

Fig. 4. Example of a pig type robot

\section{b. Wheel type robots}

This is one of the basic types of robots, which is much like a normal mobile robot. This type of robot it's used only in horizontal pipelines (Figure 5).
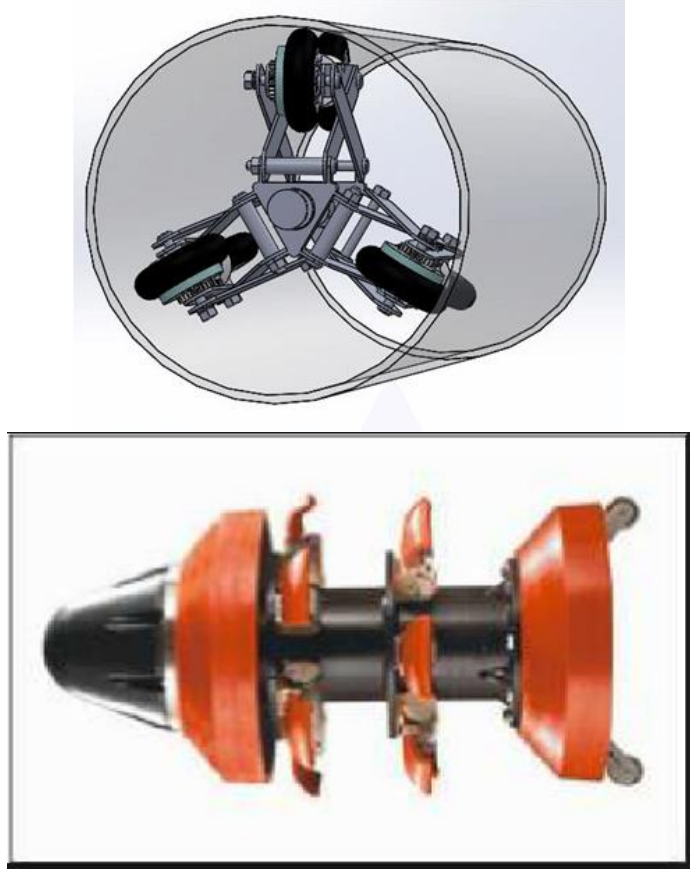

Fig. 5. Examples of wheel type robot [10]

\section{c. Caterpillar-type robots}

To enhance the contact between the robot and the pipe, a good solution could be the use of a caterpillar. This one is equipped with special made track wheel and is able to move and also climb in pipelines. The track wheel can be used to move, climb and maneuver the robot in the pipeline smoothly.

\section{d. Wall -press robots}

This is another sort of robot very useful for the vertical pipelines, which require some abilities to move in a pipe and to pass through the dividers of the pipe. The benefits of the robot are a good distribution of the robot mass, with adaptable system for all kind of weight (Figure 6).

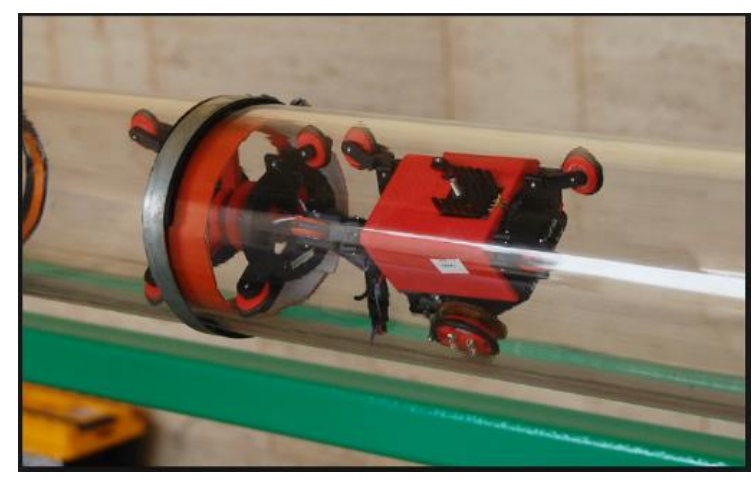

Fig. 6. Example of Wall-press robot 


\section{e. Inch worm type robots}

This robot copies the mobility of the worm, so the movement can be adapted to small diameter pipes, or in the case of diameter variations (Figure 7).

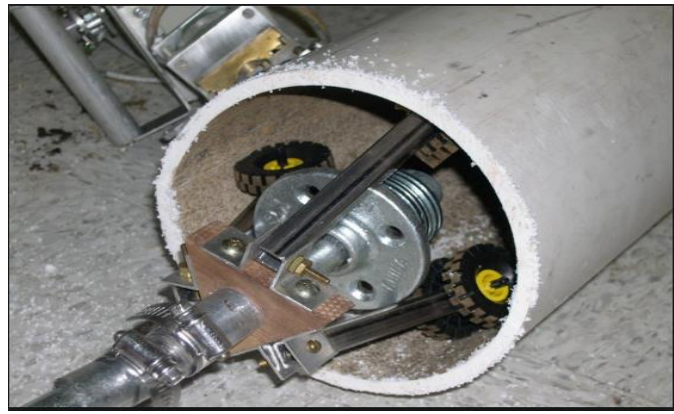

Fig. 7. Example of an inch worm type robot

\section{f. Screw type robots (helical type robots)}

This is named after the motion of this robot. This kind of robot is flexible and it has a simple structure. Its rolling motion doesn't damage the inner wall of a pipe. Therefore it could be used to inspect flexible pipes with small diameters.

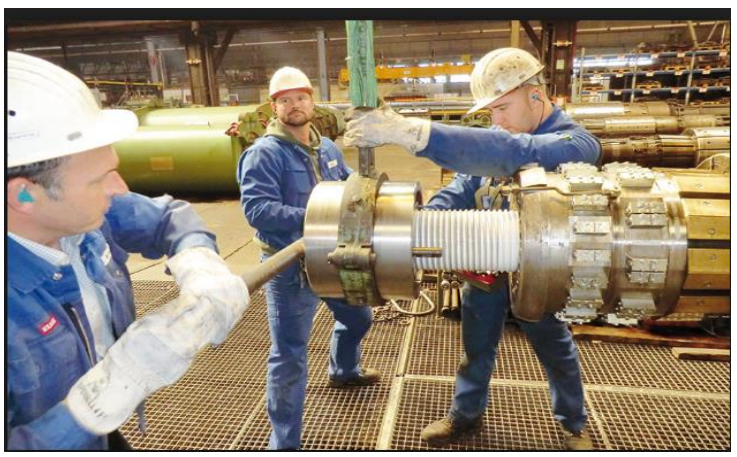

Fig. 8. Example of a helical type robot

\section{Pipelines}

Pipeline transport is the transportation of products or material through a pipe. The most recent information, in 2014, gives an aggregate of somewhat under 3.5 million $\mathrm{km}$ of pipeline in 120 nations of the world. The United States had 65\%, Russia had 8\%, and Canada had 3\%, in this way 75\% of all pipeline was in three nations [4].

Pipeline and Gas Journal's overall review figures show that 118,623 miles $(190,905 \mathrm{~km})$ of pipelines are arranged and under development. Of these, 88,976 miles $(143,193 \mathrm{~km})$ are in the arranging and configuration stage; 29,647 miles $(47,712 \mathrm{~km})$ reflect pipelines in different phases of development. Fluids and gasses are transported in pipelines and any artificially stable substance can be sent through a pipeline. Pipelines exist for the transport of fluids, for example: oil, gaseous petrol, and bio-fuels - but also for other kind of different liquids including sewage, slurry, water, and brew. Pipelines are valuable for transporting water for drinking or water system over long separations when it needs to move over slopes, or where waterways or channels are now good decisions because of vanishing, contamination, or ecological effect.

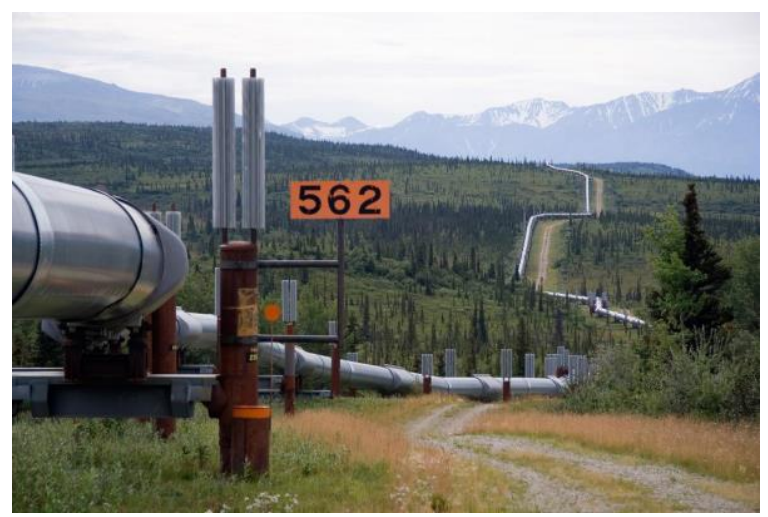

Fig. 9. Example of a pipeline: The Trans-Alaska

Pipeline in the US. Image: Wikimedia commons

The oil is transported by pipeline from the pumping along the pipeline stations lines. The situation in similar for pack flammable gas (vaporous fuel comparative) to gently known as gaseous petrol fluids (NGL). Alkali, the substance that forms a salt when mixed with an acid, is the riskiest material to be transported through pipelines over long separations. Pipeline that transport combustible materials, flammable gas or oil, raises extraordinary wellbeing concerns, and there were numerous mischance. Tubes can be the objective of vandalism, or even psychological oppressor assaults. In a war, pipelines are usually target for military assaults [5]. Oil channels of steel or plastic are typically made with the internal size of 4 to 48 inches (100 to $1220 \mathrm{~mm}$ ).

The vast majority of oil pipelines are buried at a depth of about 0.91 to $1.83 \mathrm{~m}$. It is very important to protect pipes from corrosion, impact and abrasion. A variety of methods are used for this purposes. Some of them are concrete coating, wood lagging, rock shield, high-density polyethylene, imported sand padding, and padding machines [6].

The transported crude oil contains different amounts of paraffin wax and in colder regions this content could drive to a wax buildup within the pipeline. These pipelines must be inspected and cleaned using pigging method, the practice of using devices known as PIG (pipeline inspection gauge) to perform maintenance operations on a pipeline. Those devices are also known as "Go-devils" or "scrapers". In order to detect anomalies in the pipe such as metal pieces caused by corrosion, dents, cracking or other mechanical damage, there are used "Smart pigs" (also known as "intelligent" pigs). These devices are introduced in a pipeline from a piglauncher station and move through the pipeline to 
another station down-stream. PIGs mission is to clean wax deposits or any other material accumulated inside the line or to inspect and to record the line status [7].

\section{Robotics for the Oil and Gas Industry}

The robots development is a big step for expanded the security and reliability for the oil and gas transportation. There are a lot of open doors for new applications in this field. The main idea is to develop different kind of robots that are capable to face all difficulties and to do all the tasks: inspection, cleaning, repairing, all in a hazardous environment, where a human inspector is very costly.

One great part of the mechanical research, for oil and gas industry, is committed to create in-pipe assessment robots (IPIRs) and tanks investigation robots (TIRs) [8].

Sensors and other IoT devices are frequently found on industrial equipment, particularly on oil and gas industry, to monitor and automate equipment that may be dangerous or difficult to operate. The companies can lower costs by handing off routine tasks to robots [9].

\section{Conclusions}

The conclusion of previous studies is that the use of robotics provides time and profit for money and effort from the workers. Robotics can be used in hazardous environments. Robotics can check the types of defects in the pipes so that the sensors must be used with advanced technology for the purpose of checking for high accuracy to inspection the defects, because the oil and gas pipelines are not replaceable.

A better solution could be a type of mobile robots for inspecting pipelines from the outside of pipe surfaces, but it has to work under hostile environments. It has to pass over obstacles on pipelines, such as flanges and T-joints and others.
It also has to move vertically along the pipeline and move to the adjacent pipeline for the inspection. One of the main benefits is that the inspection could be done without stopping the oil or gas transportation.

\section{References}

[1] Hashim, A.S. et al.: "Application of Robotics in Oil and Gas Refineries", International Journal of Mechanical Engineering and Technology (IJMET), 15: 01-08, (2014)

[2] "Robotics". Oxford Dictionaries. Retrieved 4 February (2011).

[3] Williams, B.: Introduction to Robotics Mechanical Engineering, Ohio University ME 4290/5290 Mechanics and Control of Robotic Manipulators, williar4@ohio.edu; (2014).

[4] "The World Factboo Central Intelligence Agency". Www.cia.gov. $\quad$ Retrieved September 6; (2016).

[5] Township, E, N.J., Pearce, J.: "George C. Devol, Inventor of Robot Arm, a suburb of Trenton. The device was used to lift and stack die-cast metal parts taken hot from their molds; (2012).

[6] Mohitpour, Mo: Pipeline Design and Construction: A Practical Approach. ASME Press. ISBN 9780791802021. (2003).

[7] Toga, Dr.S, Agarwa S.: Development and Application of Pipe Inspection Robot: A Review, Discovery; 52(246): 1210-1216; (2016).

[8] Shukla, A. and Karki, H.: A Review of Robotics In Onshore Oil-Gas Industry, Department of Mechanical Engineering Proceedings of IEEE, International Conference on Mechatronics and Automation, August 4 - 7, Takamatsu, Japan; (2013).

[9] http://www.businessinsider.com/more-robotsbeing-used-in-oil-gas-industry-2017-1

[10] Tătar, M.O., Pop, A.: Development of an in pipe inspection mini robot, Technical University of Cluj-Napoca-Romania, Faculty of Mechanics, IOP Publishing; (2016). 